\title{
First law of thermodynamics in IR Modified Hŏrava-Lifshitz gravity
}

\author{
Mengjie Wang, Jiliang Jing*, Chikun Ding, and Songbai Chen \\ Institute of Physics and Department of Physics, \\ Hunan Normal University, Changsha, \\ Hunan 410081, P. R. China \\ and \\ Key Laboratory of Low Dimensional Quantum Structures \\ and Quantum Control of Ministry of Education, \\ Hunan Normal University, Changsha, \\ Hunan 410081, P.R. China
}

\begin{abstract}
We study the first law of thermodynamics in IR modified Horrava-Lifshitz spacetime. Based on the Bekenstein-Hawking entropy, we obtain the integral formula and the differential formula of the first law of thermodynamics for the Kehagias-Sfetsos black hole by treating $\omega$ as a new state parameter and redefining a mass that is just equal to $M_{A D M}$ obtained by Myung[33] if we take $\alpha=3 \pi / 8$.

PACS numbers: $95.30 . \mathrm{Tg}, 04.70 .-\mathrm{s}$, 97.60.Lf
\end{abstract}

\footnotetext{
* Corresponding author, Electronic address: jljing@hunnu.edu.cn
} 


\section{INTRODUCTION}

Many researchers are focused on black hole physics, and many significant and interesting results have been achieved, including Hawking radiation, black hole thermodynamics, and so on. In 1973, Bardeen, Cater, and Hawking found that the integral formula for the first law of black hole mechanics for a stationary axisymmetric asymptotically flat black hole is given by [1]

$$
M=\frac{\kappa}{4 \pi} A+2 \Omega_{H} J_{H}+\frac{1}{4 \pi} \int_{S} R_{a}^{b} \xi_{(t)}^{a} d \Sigma_{b},
$$

where $\kappa, A, \Omega_{H}, J_{H}, R_{a}^{b}, \xi_{(t)}^{a}, d \Sigma_{b}$ are the surface gravity at the event horizon, the area of the event horizon, angular velocity, angular momentum, Ricci tensor, timelike Killing vector, and surface element, respectively. Using Eq.(1.1), they obtained the differential formula for the first law of black hole mechanics [1]

$$
\delta M=\frac{\kappa}{8 \pi} \delta A+\Omega_{H} \delta J_{H}+\int \Omega \delta d J+\int \bar{\mu} \delta d N+\int \bar{\theta} \delta d S,
$$

where $\delta d N$ is the change in the number of particles crossing $d \Sigma_{b}, \delta d S$ is the change in the entropy crossing $d \Sigma_{b}, \bar{\mu}$ is the "redshifted "chemical potential and $\bar{\theta}$ is the red-shifted temperature. Then Bekenstein [2] introduce the concept of thermodynamics into black hole physics and Hawking [3] proved that the black hole is indeed not entirely black and emits radiation by using the quantum fields theory in curved spacetime. Thus, the temperature and entropy of black holes are given by

$$
T=\frac{\kappa}{2 \pi}, \quad S=\frac{A}{4} .
$$

Recently, Hǒrava [4 6] proposed a new class of quantum gravity that is nonrelativistic and power-counting renormalizable. It is a theory with higher spatial derivatives, and the key property of this theory is the three-dimensional general covariance and time reparameterization invariance. It is this anisotropic rescaling that makes Horava's theory power-counting renormalizable. Therefore, a lot of attention has been focused on this theory of gravity, and its cosmological applications have been studied [8 15]. Some static spherically symmetric black hole solutions have been found in Hořava's theory [16 22]. The general IR vacuum has a nonzero cosmological constant in Hořava's theory[7]. In order to get a Minkowsky vacuum in the IR region, one must add a new term $\mu^{4} R^{(3)}$ in the action and take the $\Lambda_{W} \rightarrow 0$ limit for the cosmological constant $\Lambda_{W}$. This does not change the UV properties of the theory, but it alters the IR properties. Making use of such a modified action, Kehagias and Sfetsos [14] obtained the asymptotic flat spherically symmetric vacuum black hole solution(KS black hole). This black 
hole behaves like the Reissner-Norström black hole and has two event horizons. Moreover, the heat capacity is positive for the small black hole, and it is negative for the large one. It means that the small black hole is stable in the Hořava's theory, which is quite different from that of the Schwarzschild solution in Einstein's theory. The investigation of the quasinormal modes of the massless scalar perturbations shows that the perturbations live longer in the IR modified Hǒrava-Lifshitz spacetime [30, 31]. These results imply that distinct differences exist between Hǒrava-Lifshitz's theory and Einstein's gravity.

Because gravity theory, quantum theory, and statistical mechanics are merged into black hole thermodynamics, it is believed that some clues on quantum effects of gravity would be revealed in black hole thermodynamics. Therefore, a lot of attention [23 27] has been focused on black hole thermodynamics for Hǒrava-Lifshitz gravity, and thermodynamics for the KS black hole was also investigated and some peculiar results were obtained [28, 29]. The general procedure for investigating KS black hole thermodynamics is to assume that the first law of thermodynamics is [28, 29]

$$
d m=T d S
$$

and the entropy is obtained as [28, 29]

$$
S=\int \frac{d m}{T}+S_{0}=\pi r_{+}^{2}+\frac{\pi}{\omega} \log \left(r_{+}^{2}\right)+S_{0}=\frac{A}{4}+\frac{\pi}{\omega} \log \left(r_{+}^{2}\right)+S_{0},
$$

with $S_{0}$ as an integration constant.

We have several comments regarding the above equations: (i) compared with the ReissnerNordström black hole, we know that Eq.(1.4) is flawed for the KS black hole, and it should be modified with a work term. (ii) It is obvious that the integral formula

$$
m=k T S
$$

is not satisfied for the KS black hole, where $k$ is a proportional constant (for example, $k=2$ for 4-dimensional Schwarzschild spacetime and $k=(d-2) /(d-3)$ for the d-dimensional Schwarzschild spacetime), and (iii) the expression for entropy of the KS black hole is not consistent with Bekenstein-Hawking entropy, $S=A / 4$.

In order to solve the above problems uniformly, motivated by [32], we find that $\omega$ in the KS black hole can be viewed as a charge in some degree. By using the entropy of the KS black hole, which is consistent with Bekenstein-Hawking entropy, and redefining a new mass, the integral formula and differential formula of the first law of thermodynamics for the KS black hole, which are compatible with each other, can be obtained.

The remainder of this paper is organized as follows: In Sec. II, we give a brief description of the solution in the IR modified Hǒrava-Lifshitz black hole spacetime. In Sec. III, the integral 
and differential form of the first law of thermodynamics for the KS black hole are presented. In Sec. IV, the statistical entropy for the KS black hole is studied. Finally, we summarize our conclusions in the last section.

\section{BLACK HOLE IN IR MODIFIED HǑRAVA-LIFSHITZ GRAVITY}

The general metric can be written in the following form in the $(3+1)$-dimensional ArnowittDeser-Misner formalism:

$$
d s^{2}=-N^{2} d t^{2}+g_{i j}\left(d x^{i}+N^{j} d t\right)\left(d x^{j}+N^{j} d t\right)
$$

and its extrinsic curvature $K_{i j}$ is

$$
K_{i j}=\frac{1}{2 N}\left(\dot{g}_{i j}-\nabla_{i} N_{j}-\nabla_{j} N_{i}\right)
$$

In the Hǒrava theory, a modified action in the IR region is given by [14]

$$
\begin{aligned}
S_{H L} & =\int d t d^{3} x\left(\mathcal{L}_{0}+\tilde{\mathcal{L}}_{1}\right), \\
\mathcal{L}_{0} & =\sqrt{g} N\left\{\frac{2}{\kappa^{2}}\left(K_{i j} K^{i j}-\lambda K^{2}\right)+\frac{\kappa^{2} \mu^{2}\left(\Lambda_{W} R^{(3)}-3 \Lambda_{W}^{2}\right)}{8(1-3 \lambda)}\right\}, \\
\tilde{\mathcal{L}}_{1} & =\sqrt{g} N\left\{\frac{\kappa^{2} \mu^{2}(1-4 \lambda)}{32(1-3 \lambda)}\left(R^{(3)}\right)^{2}-\frac{\kappa^{2}}{2 w^{4}}\left(C_{i j}-\frac{\mu w^{2}}{2} R_{i j}^{(3)}\right)\left(C^{i j}-\frac{\mu w^{2}}{2} R^{(3) i j}\right)+\mu^{4} R^{(3)}\right\},
\end{aligned}
$$

where $\kappa^{2}, \lambda, \mu, w$, and $\Lambda_{W}$ are constant parameters, $R^{(3)}$ and $R_{i j}^{(3)}$ are three-dimensional spatial Ricci scalar and Ricci tensor. The Cotton tensor $C_{i j}$ is

$$
C^{i j}=\epsilon^{i k \ell} \nabla_{k}\left(R_{\ell}^{(3) j}-\frac{1}{4} R^{(3)} \delta_{\ell}^{j}\right) .
$$

Taking the $\Lambda_{W} \rightarrow 0$ limit and letting $\lambda=1$, it was found that the speed of light and the Newton constant are described by the following relations [14]:

$$
c^{2}=\frac{\kappa^{2} \mu^{4}}{2}, \quad G=\frac{\kappa^{2}}{32 \pi c} .
$$

Considering a static and spherically symmetric background as

$$
d s^{2}=-N^{2}(r) d t^{2}+\frac{d r^{2}}{f(r)}+r^{2}\left(d \theta^{2}+\sin ^{2} \theta d \phi^{2}\right)
$$

Eq.(2.4) is changed into

$$
\tilde{\mathcal{L}}_{1}=\sqrt{g} N\left\{\frac{3 \kappa^{2} \mu^{2}}{64}\left(R^{(3)}\right)^{2}-\frac{\kappa^{2} \mu^{2}}{8} R^{(3) i j} R_{i j}^{(3)}+\mu^{4} R^{(3)}\right\},
$$


where

$$
R^{(3) i j} R_{i j}^{(3)}=\frac{f^{\prime}(r)^{2}}{r^{2}}+\frac{\left(-2+2 f(r)+r f^{\prime}(r)\right)^{2}}{2 r^{4}}, \quad R^{(3)}=-\frac{2\left(-1+r f(r)+f^{\prime}(r)\right)}{r^{2}} .
$$

Varying the action with $\mathrm{N}(\mathrm{r})$ and $\mathrm{f}(\mathrm{r})$ respectively, we then obtain the KS black hole solution[14]

$$
N^{2}(r)=f(r)=1+\omega r^{2}-\sqrt{\omega^{2} r^{4}+4 \omega m r},
$$

where $m$ is an integration constant related to the black hole mass.

The parameter $m$ can be expressed by horizon radius $r_{+}$as

$$
m=\frac{1+2 \omega r_{+}^{2}}{4 \omega r_{+}} .
$$

The outer and inner event horizon are given by

$$
r_{+}=m+\sqrt{m^{2}-\frac{1}{2 \omega}}, \quad r_{-}=m-\sqrt{m^{2}-\frac{1}{2 \omega}},
$$

with $m^{2} \geq \frac{1}{2 \omega}$, and the extremal black hole should be satisfied $m^{2}=\frac{1}{2 \omega}$. The surface gravity is

$$
\kappa=\frac{f^{\prime}\left(r_{+}\right)}{2}=\frac{2 \omega r_{+}^{2}-1}{4 r_{+}\left(1+\omega r_{+}^{2}\right)}
$$

and the corresponding temperature is given by

$$
T=\frac{\kappa}{2 \pi}=\frac{2 \omega r_{+}^{2}-1}{8 \pi r_{+}\left(1+\omega r_{+}^{2}\right)} .
$$

\section{FIRST LAW OF THE KS BLACK HOLE}

For an asymptotically flat black hole, Bardeen, Cater and Hawking [1] gave a differential geometric method to calculate the integral formula and differential formula of black hole mechanics. Because the KS black hole is an asymptotically flat black hole, we will follow Bardeen, Cater and Hawking [1] to obtain the integral and differential formula for the KS black hole.

According to Eq.(2.10), we know that there exist two Killing vector fields, i.e., the timelike Killing vector field and the spacelike Killing vector field, which are denoted by $\xi_{(t)}$ and $\xi_{(\varphi)}$, respectively. These Killing vector fields obey equations [1]

$$
\begin{gathered}
\nabla_{b} \xi_{(t) a}=\nabla_{[b} \xi_{(t) a]}, \quad \nabla_{b} \xi_{(\varphi) a}=\nabla_{[b} \xi_{(\varphi) a]} \\
\xi_{(\varphi)}^{b} \nabla_{b} \xi_{(t) a}=\xi_{(t)}^{b} \nabla_{b} \xi_{(\varphi) a} \\
\nabla^{b} \xi_{(t) b}^{a}=-R_{b}^{a} \xi_{(t)}^{b}
\end{gathered}
$$




$$
\nabla^{b} \xi_{(\varphi) b}^{a}=-R_{b}^{a} \xi_{(\varphi)}^{b},
$$

where $\nabla_{a}$ represents a covariant derivative, and the square brackets around indices imply antisymmetrization.

One can integrate Eq.(3.3) over a hypersurface $\mathrm{S}$ and transfer the volume on the left to an integral over a 2-surface $\partial S$ bounding $S$

$$
\int_{\partial S} \nabla^{b} \xi_{(t)}^{a} d \Sigma_{a b}=-\int_{S} R_{b}^{a} \xi_{(t)}^{b} d \Sigma_{a},
$$

where $d \Sigma_{a b}$ and $d \Sigma_{a}$ are the surface elements of $\partial S$ and $S$, respectively. The boundary $\partial S$ of $S$ consists of $\partial S_{B}$ and a 2-surface $\partial S_{\infty}$ at infinity. Calculating the left expression in Eq.(3.5) at infinity, we have

$$
\int_{\partial S_{\infty}} \nabla^{b} \xi_{(t)}^{a} d \Sigma_{a b}=-4 \pi m
$$

where $\mathrm{m}$ is the mass as measured from infinity.

Substituting Eq.(3.6) into Eq.(3.5), we have

$$
m=\frac{1}{4 \pi} \int_{\partial S_{B}} \nabla^{b} \xi_{(t)}^{a} d \Sigma_{a b}+\frac{1}{4 \pi} \int_{S} R_{b}^{a} \xi_{(t)}^{b} d \Sigma_{a} .
$$

Introducing the null vector $l^{a}$, which is equivalent to the timelike Killing vector in our case

$$
l^{a}=\xi_{(t)}^{a},
$$

using the surface gravity

$$
\kappa=l_{a ; b} l^{a} n^{b},
$$

and the surface element of the event horizon $d \Sigma_{a b}$

$$
d \Sigma_{a b}=l_{[a} n_{b]} d A,
$$

Eq.(3.7) can be rewritten as

$$
m=\frac{\kappa A}{4 \pi}+\frac{1}{4 \pi} \int_{S} R_{b}^{a} \xi_{(t)}^{b} d \Sigma_{a} .
$$

Because $\xi_{(t)}$ is a timelike Killing vector, we let $\xi_{(t)}=(1,0,0,0)$ for the KS black hole. Therefore, the second term in the right side of Eq.(3.11) can be expressed as

$$
\frac{1}{4 \pi} \int_{S} R_{b}^{a} \xi_{(t)}^{b} d \Sigma_{a}=\frac{1}{4 \pi} \int_{S} R_{0}^{0} \xi_{(t)}^{0} d \Sigma_{0},
$$


with

$$
R_{0}^{0}=-\frac{3 \omega^{2}\left(2 m^{2}+6 \omega m r^{3}+\omega^{2} r^{6}-4 m r \sqrt{\omega^{2} r^{4}+4 \omega m r}-\omega r^{4} \sqrt{\omega^{2} r^{4}+4 \omega m r}\right)}{\left(\omega^{2} r^{4}+4 \omega m r\right)^{\frac{3}{2}}} .
$$

Substituting Eq.(3.13) into Eq.(3.12), we get

$$
\frac{1}{4 \pi} \int_{S} R_{b}^{a} \xi_{(t)}^{b} d \Sigma_{a}=\frac{1+4 \omega r_{+}^{2}}{4 \omega r_{+}\left(1+\omega r_{+}^{2}\right)}
$$

Then substituting Eq.(3.14) into Eq.(3.11), and considering Eq.(1.3), we have

$$
m=2 T S+\frac{1+4 \omega r_{+}^{2}}{4 \omega r_{+}\left(1+\omega r_{+}^{2}\right)} .
$$

Comparing Eq.(2.12) with the outer and inner event horizon of the Reissner-Nordström black hole

$$
r_{+}=m+\sqrt{m^{2}-Q^{2}}, \quad r_{-}=m-\sqrt{m^{2}-Q^{2}}
$$

and motivated by [32], it is obvious that $\frac{1}{2 \omega}$ is equivalent to $Q^{2}$, and this means that we could view $\frac{1}{2 \omega}$ as a charge in some degree. Therefore, we can formally recast Eq.(3.15) into

$$
M=2 T S+V \frac{1}{\sqrt{2 \omega}}
$$

where $M$ is a new mass, and $V$ is the potential corresponding to $\frac{1}{\sqrt{2 \omega}}$. At the same time, the differential formula of the first law should be taken in the following form:

$$
d M=T d S+V d\left(\frac{1}{\sqrt{2 \omega}}\right) .
$$

According to the exact differential condition

$$
\frac{\partial}{\partial \omega}\left(2 \pi T r_{+}\right)=\frac{\partial}{\partial r_{+}}\left(-\frac{V}{2 \sqrt{2} \omega^{\frac{3}{2}}}\right)
$$

and using Eqs.(3.18) and (2.14), we get the expressions for $M$ and $V$

$$
\begin{gathered}
M=\frac{r_{+}}{2}-\frac{3}{4 \sqrt{\omega}} \arctan \left(r_{+} \sqrt{\omega}\right)+h(\omega), \\
V=-\frac{3\left(\left(1+\omega r_{+}^{2}\right) \arctan \left(\sqrt{\omega} r_{+}\right)-\sqrt{\omega} r_{+}\right)}{2 \sqrt{2}\left(1+\omega r_{+}^{2}\right)}-g(\omega),
\end{gathered}
$$

where $g(\omega)$ and $h(\omega)$ are two integration parameters. The relation between them is confined by Eq.(3.18), i.e.,

$$
h^{\prime}(\omega)=\frac{g(\omega)}{2 \sqrt{2} \omega^{\frac{3}{2}}} .
$$


Substituting Eqs. (3.20) and (3.21) into Eq. (3.17) and together with Eq.(3.22), we obtain

$$
h(\omega)=\frac{\alpha}{\sqrt{\omega}}, \quad g(\omega)=-\sqrt{2} \alpha
$$

where $\alpha$ is an integration constant.

Then the expressions for $M$ and $V$ from Eqs. (3.20), (3.21) and (3.23) are

$$
\begin{gathered}
M=\frac{r_{+}}{2}-\frac{3}{4 \sqrt{\omega}} \arctan \left(r_{+} \sqrt{\omega}\right)+\frac{\alpha}{\sqrt{\omega}} \\
V=\frac{3 r_{+} \sqrt{\omega}+4 \alpha\left(1+\omega r_{+}^{2}\right)-3\left(1+\omega r_{+}^{2}\right) \arctan \left(\sqrt{\omega} r_{+}\right)}{2 \sqrt{2}\left(1+\omega r_{+}^{2}\right)} .
\end{gathered}
$$

We should note that $\alpha>\frac{3}{4} \arctan \frac{1}{\sqrt{2}}-\frac{1}{2 \sqrt{2}}$ to keep $M$ is positive. We also note that the expression for $M$ in Eq.(3.24) is the same as the mass $M_{A D M}$ in Ref. [33] if we take $\alpha=\frac{3 \pi}{8}$.

The above discussions show that the integral and differential formula of the first law of thermodynamics for the KS black hole can be expressed as Eqs.(3.17) and (3.18), and the expressions for $M$ and $V$ are given by Eqs.(3.24) and (3.25).

\section{STATISTICAL ENTROPY FOR THE KS BLACK HOLE}

The purpose of this section is to demonstrate that Bekenstein-Hawking entropy also exists for the KS black hole. If we recover $\hbar$, Eq.(1.5) is changed into

$$
S=\int \frac{d m}{T}+S_{0}=\frac{\pi r_{+}^{2}}{\hbar}+\frac{\pi}{\omega \hbar} \log \left(r_{+}^{2}\right)+S_{0}=\frac{A}{4 \hbar}+\frac{\pi}{\omega \hbar} \log \left(r_{+}^{2}\right)+S_{0} .
$$

It is obvious that both the Bekenstein-Hawking term and the logarithmic term have the same order of $\hbar$; therefore, it is inappropriate for one to view the logarithmic term as a higher order quantum correction.

In what follows, we strictly prove that the entropy obtained from Eq.(4.1) is incorrect and the Bekenstein-Hawking entropy, $S=A / 4$, also holds for the KS black hole by using the thin

film brick wall model, provided that only the order of $\hbar^{-1}$ is considered. For simplicity, we only consider the massless case.

For massless particle, we have $P_{\mu} P^{\mu}=0$, i.e.,

$$
g^{t t} P_{t}^{2}+g^{r r} P_{r}^{2}+g^{\theta \theta} P_{\theta}^{2}+g^{\varphi \varphi} P_{\varphi}^{2}=0
$$

The module of the spatial component of the four-momentum is

$$
P^{2} \equiv P_{j} P^{j}=g^{r r} P_{r}^{2}+g^{\theta \theta} P_{\theta}^{2}+g^{\varphi \varphi} P_{\varphi}^{2}=-g^{t t} P_{t}^{2},
$$


and the number of the quantum state is

$$
\Gamma=\frac{1}{(2 \pi \hbar)^{3}} \int d r d \theta d \varphi d P_{r} d P_{\theta} d P_{\varphi}
$$

For convenience, we set $P_{1}^{2}=g^{r r} P_{r}^{2}, P_{2}^{2}=g^{\theta \theta} P_{\theta}^{2}, P_{3}^{2}=g^{\varphi \varphi} P_{\varphi}^{2}$, and take $P^{2}=P_{1}^{2}+P_{2}^{2}+P_{3}^{2}$. Then, Eq. (4.4) becomes

$$
\begin{aligned}
\Gamma & =\frac{1}{(2 \pi \hbar)^{3}} \int d r d \theta d \varphi \frac{1}{\sqrt{g^{r r} g^{\theta \theta} g^{\varphi \varphi}}} \frac{4}{3} \pi P^{3} \\
& =\frac{1}{6 \pi^{2} \hbar^{3}} \int \sqrt{g_{\theta \theta} g_{\varphi \varphi} g_{r r}} d r d \theta d \varphi\left(-g^{t t} P_{t}^{2}\right)^{\frac{3}{2}} \\
& =\frac{\left(-P_{t}\right)^{3}}{6 \pi^{2} \hbar^{3}} \int \frac{\sqrt{-D e t}}{g_{t t}^{2}} d r d \theta d \varphi,
\end{aligned}
$$

where $\sqrt{-D e t}=\sqrt{-g_{t t} g_{r r} g_{\theta \theta} g_{\varphi \varphi}}$. The free energy is then given by

$$
\begin{aligned}
F(\beta) & =\frac{1}{\beta} \int d \Gamma \ln \left(1-e^{-\beta \omega}\right) \\
& =-\int_{0}^{\infty} \frac{\Gamma}{e^{\beta \omega}-1} d \omega \\
& =-\frac{1}{6 \pi^{2} \hbar^{3}} \int_{0}^{\infty} \frac{\left(-P_{t}\right)^{3}}{e^{\beta \omega}-1} d \omega \int_{r_{+}+\epsilon}^{r_{+}+\epsilon+\delta} \frac{\sqrt{-D e t}}{g_{t t}^{2}} d r d \theta d \varphi \\
& =-\frac{2 \pi^{3}}{45 \beta^{4}} \int_{r_{+}+\epsilon}^{r_{+}+\epsilon+\delta} \frac{r^{2}}{g_{t t}^{2}} d r .
\end{aligned}
$$

From which we can get the entropy of the KS black hole

$$
S=\frac{8 \pi^{3}}{45 \beta(4 \pi)^{2}} \frac{r_{+}^{2} \delta}{\epsilon(\epsilon+\delta)}=\frac{\pi r_{+}^{2}}{90 \beta} \frac{\delta}{\epsilon(\epsilon+\delta)}=\frac{A}{4},
$$

where we let $\frac{\delta}{\epsilon(\epsilon+\delta)}=90 \beta$. Equation (4.7) implies that the semiclassical entropy for the KS black hole satisfies the Bekenstein-Hawking entropy.

\section{CONCLUSION}

We have studied the first law of thermodynamics for the KS black hole. If we assume that the differential formula of the first law of thermodynamics is $d m=T d S$, some unsatisfactory results occur, i.e., the Bekenstein-Hawking entropy and the integral formula of the first law of thermodynamics do not hold. By analogy with the Reissner-Nordström black hole, we know that Eq.(1.4) is flawed for the KS black hole and it should be modified with a work term. Based on Bekenstein-Hawking entropy, following the method provided in Ref. [1], we obtain the integral formula (3.17) and the differential formula (3.18) of the first law of thermodynamics for the KS black hole by treating $\omega$ as a new state parameter and redefining a new mass (3.24). The new mass is just equal to $M_{A D M}$ in Ref. [33], if we take $\alpha=3 \pi / 8$. 


\section{Acknowledgments}

This work was supported by the National Natural Science Foundation of China under Grant No. 10875040, the key project of the National Natural Science Foundation of China under Grant No 10935013, the National Basic Research of China under Grant No. 2010CB833004, the Hunan Provincial Natural Science Foundation of China under Grant No. 08JJ3010, and the Construct Program of the National Key Discipline. S. B. Chen's work was partially supported by the National Natural Science Foundation of China under Grant No.10875041; the Scientific Research Fund of Hunan Provincial Education Department Grant No.07B043.

[1] Bardeen J M, Cater B, Hawking S W, Commun Math Phys 31 (1973) 161.

[2] Jacob D. Bekenstein, Phys. Rev. D 7, 2333 (1973).

[3] Hawking S W, Math.Phys. 43, 199 (1975).

[4] P. Horava, Phys. Rev. D 79084008 (2009).

[5] P. Horava, JHEP 03, 020 (2009).

[6] P. Hořava, Phys. Rev. Lett. 102, 161301 (2009).

[7] T. P. Sotiriou, M. Visser and S. Weinfurtner, Phys. Rev. Lett. 102, 251601 (2009).

[8] G. Calcagni, JHEP 09, 112 (2009).

[9] Remo Garattini, arXiv: 0912.0136 [gr-qc].

[10] Xian Gao, Yi Wang, Wei Xue, Robert Brandenberger, J. Cosmol. Astropart. Phys. 02 (2010) 020 .

[11] S. Dutta, E. N. Saridakis , J. Cosmol. Astropart. Phys. 01 (2010) 013.

[12] Y. S. Piao, Phys Lett B 681, 1 (2009).

[13] X. Gao, arXiv: 0904.4187 [hep-th].

[14] A. Kehagias and K. Sfetsos, Phys Lett B 678, 123 (2009).

[15] E. Kiritsis and G. Kofinas, Nucl. Phys. B 821, 467 (2009).

[16] H. Lu, J. Mei and C. N. Pope, Phys.Rev.Lett. 103, 091301 (2009).

[17] Jin-Zhang Tang, arXiv: 0911.3849 [hep-th].

[18] E. Kiritsis , Phys. Rev. D 81, 044009 (2010).

[19] M. R. Setare, D. Momeni , arXiv: 0911.1877 [hep-th].

[20] R. G. Cai, L. M. Cao and N. Ohta, Phys. Rev. D 80, 024003 (2009). 
[21] E. O. Colgain and H. Yavartanoo, JHEP 08, 021 (2009).

[22] D. Capasso, A. P. Polychronakos, arXiv: 0911.1535 [hep-th].

[23] R. G. Cai, N. Ohta, arXiv: 0910.2307 [hep-th].

[24] Jun-Jin Peng, Shuang-Qing Wu, Eur. Phys. J. C (2010) 66: 325-331.

[25] Anzhong Wang, Yumei Wu , J. Cosmol. Astropart. Phys. 07, 012 (2009).

[26] Y. S. Myung and Y. W. Kim, arXiv: 0905.0179 [hep-th].

[27] R. G. Cai, L. M. Cao and N. Ohta, Phys.Lett.B 679, 504 (2009).

[28] Y. S. Myung Phys. Lett. B 684, 158 (2010).

[29] Andres Castillo, Alexis Larranaga. arXiv: 0906.4380 [gr-qc].

[30] S. B. Chen, J. L. Jing, arXiv:0905.1409 [hep-th].

[31] R. A. Konoplya. Phys.Lett.B 679, 499 (2009).

[32] Y. Sekiwa, Phys.Rev. D 73, 084009 (2006).

[33] Yun Soo Myung, Phys.Lett.B 685: 318, 2010. 